\title{
Linear plication and external wrapping in ascending aortic aneurysm in well-selected patients: Surgical technique
}

Mohammad Alşalaldeh ${ }^{1 *}$, Ali Vefa Özcan ${ }^{1 a}$

${ }^{1}$ Pamukkale University, Faculty of Medicine Hospital, Cardiovascular Surgery - Denizli / TURKEY

*Corresponding Author: Mohammad Alşalaldeh, Pamukkale University, Faculty of Medicine Hospital, Cardiovascular Surgery - Denizli / TURKEY

Received Date: April 01, 2021; Accepted Date: April 10, 2021; Published Date; April 22, 2021

Citation: Alşalaldeh M*, Ali V Özcan. (2021) Linear plication and external wrapping in ascending aortic aneurysm in well-selected patients: Surgical technique. J. Surg Case Repo and Imag. 4(3); DOI:10.31579/2690-1897/069

Copyright: (C) 2021 Mohammad Alşalaldeh, This is an open-access article distributed under the terms of the Creative Commons Attribution License, which permits unrestricted use, distribution, and reproduction in any medium, provided the original author and source are credited

\begin{abstract}
An aortic aneurysm is known as a bulging or dilatation of the aorta for more than one and half of its normal diameter result in making the wall of the aorta thinner and at the risk of dissection or rupture. For a long time ago surgeons have worked hard to find the best surgical technique to treat this pathology. Open surgery with the replacement of the aneurysmatic segment by a synthetic graft is still the standard surgical treatment method.

In the past, some surgeons tried patching the aneurysmatic segment with synthetic patches. This technique was not accepted by many surgeons. In our cardiovascular surgical center, we perform linear plication and external wrapping (LPEW) surgical technique for more than ten years in well-selected patients whose aortic aneurysm diameter is less than $6 \mathrm{~cm}$ with normal aortic root and who has not any connective tissue disease, dissection, or rupture. Our results are satisfying with low morbidity and mortality.

Keywords: Ascending aortic aneurysm, aortic dissection, linear plication and external wrapping, dacron graft replacement, aortic dissection, revision.
\end{abstract}

\section{Introduction:}

Ascending aortic aneurysm is one of the aortic pathologies that carries high mortality and morbidity mainly when the aneurysmatic aorta has dissection or rupture. Open surgical treatment can be achieved by replacement as well as it is done worldwide or by other alternative surgical methods [. In our center, we have good experience in managing such cases in well-selected patients by linear plication and external wrapping (LPEW). Here, we will describe this surgical technique [1].

\section{Technique Description}

After all pre-operation preparations have been completed the patient is taken to the operation room and the operation is started under general anesthesia. If the patient has concomitant another heart disease such as coronary artery disease or valvular disease, those pathologies are treated first with coronary artery bypass grafting or valvular repair or replacement. Cross clamp released from the ascending aorta and LPEW started on beating heart. Firstly, the ascending aorta is well liberated from the surrounding tissues.

A 30-32 mm Dacron graft with suitable length is prepared by cutting it longitudinally after bringing it into a trapezoid shape (Figure 1). Then the graft is passed under the aorta to let its short curvature sit on the upper border of the aorta. A long side vascular clamp is applied longitudinally at the right anterolateral side of the ascending aorta starting from above sinüs Valsalva to the beginning of the innominate artery and giving an acceptable diameter size to the remaining aorta. The two edges of the graft are sewn underneath the straight clamp through the plicated zone in ' $U$ ' shape suturing style by using 4/0 polypropylene sutures [2]. A straight clamp is released (Figure 2). 

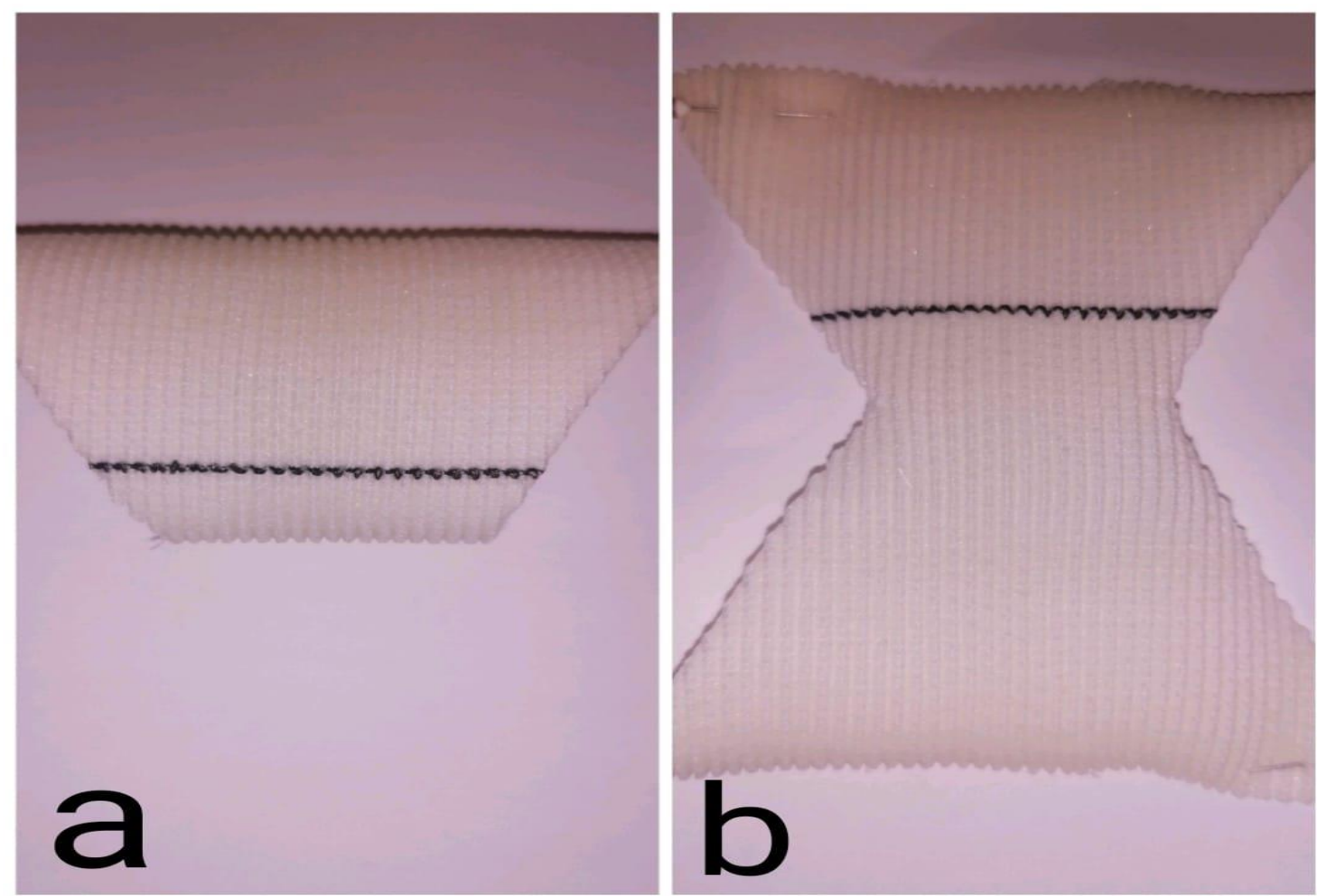

Figure 1: a) Dacron graft is prepared by cutting the two edges oblique to give it a trapezoid shape. b) Then it is cut longitudinally from its long curvature.

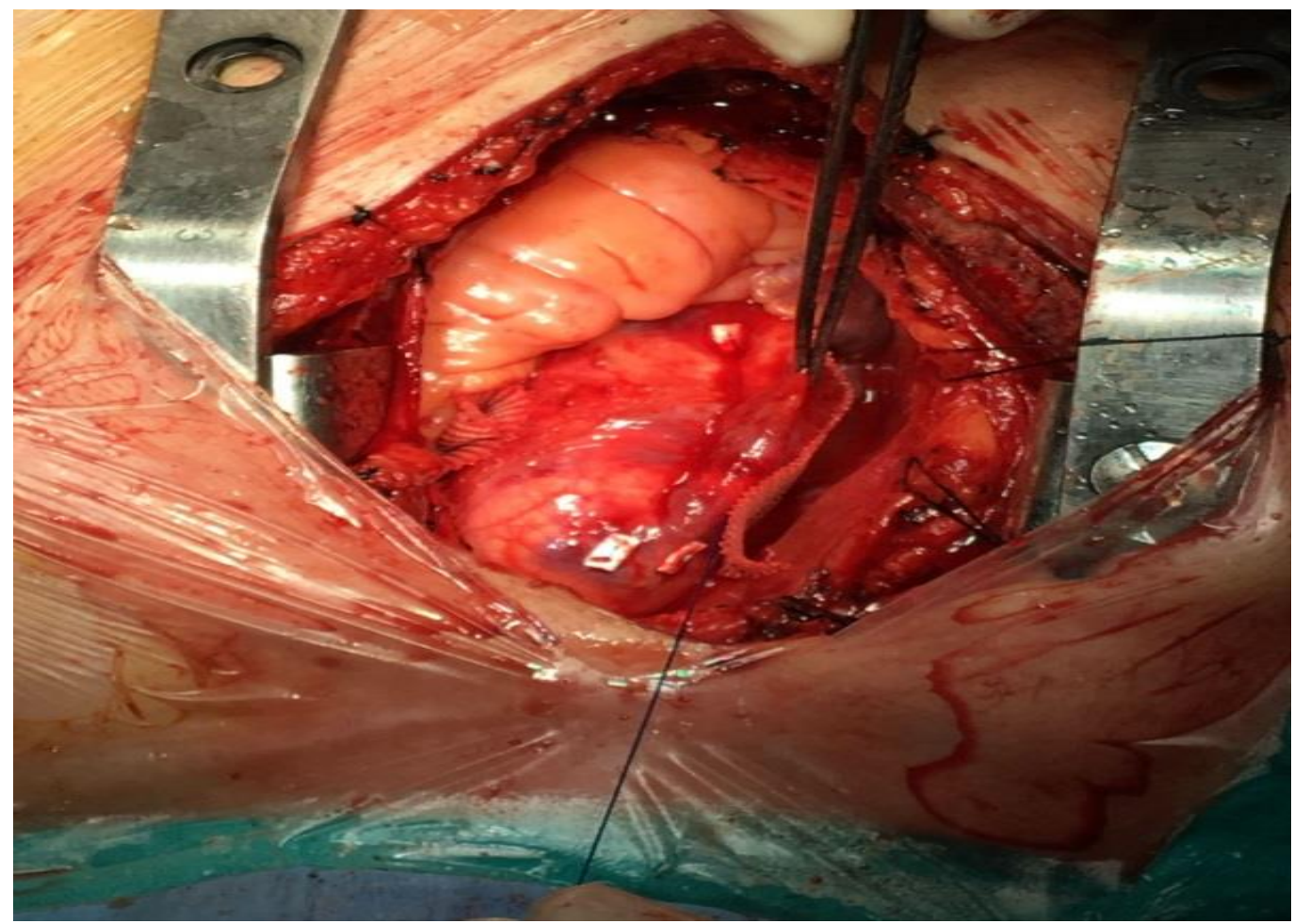

Figure 2: The two edges of the graft are sewn underneath the straight clamp through the plicated zone in 'U' shape suturing style.

A 2/0 polyester suture is used in suturing the graft edges with the plication zone in continuous style. The proximal and distal edges of the graft are fixated to the anterior adventitia of the aorta by $5 / 0$ polyproline sutures to prevent graft immigration (Figure 3). In CABG cases the graft is prepared previously to allow the passage of the proximal graft through it. Then decannulation is done traditionally. 


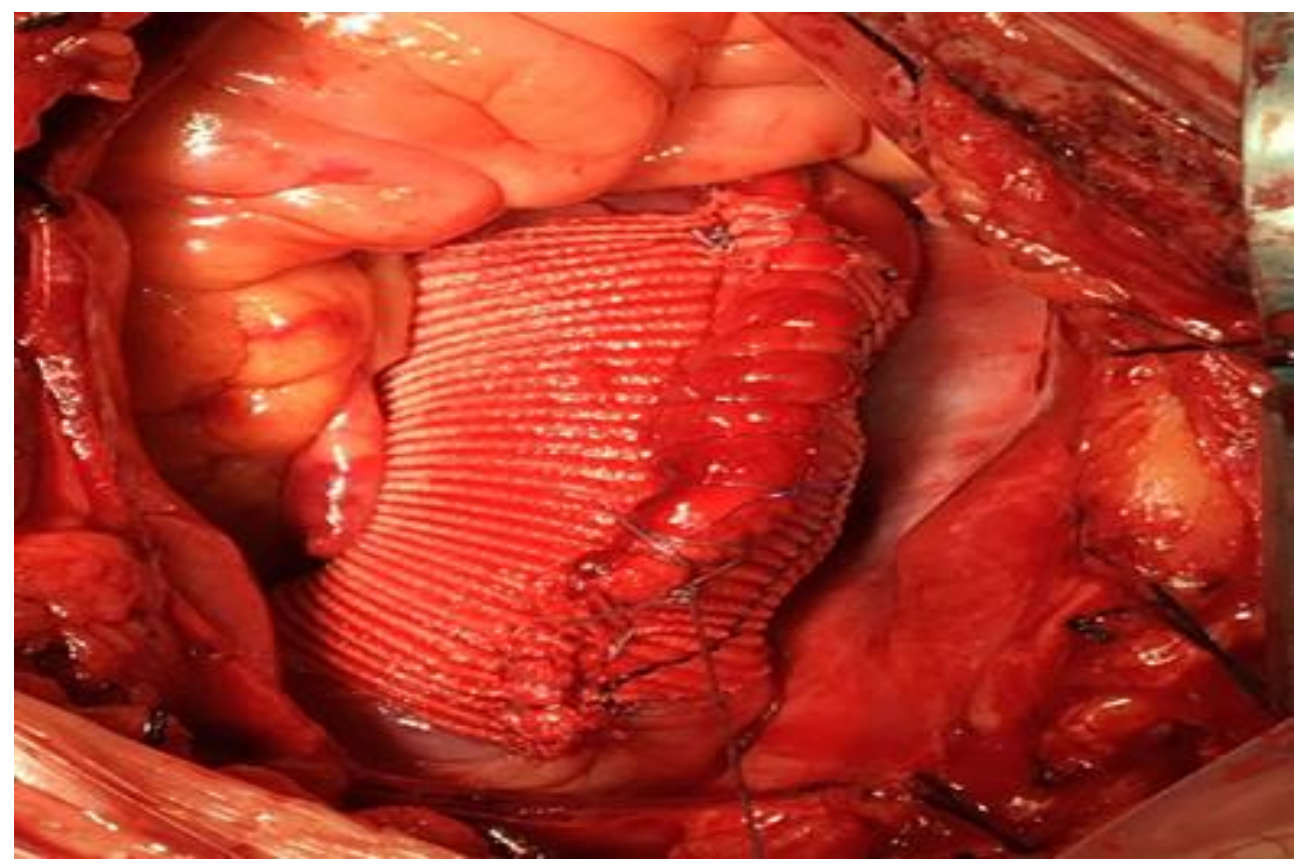

Figure 3: The proximal and distal edges of the graft are fixated to the anterior adventitia of the aorta by $5 / 0$ polyproline sutures to prevent graft immigration

\section{Discussion}

Ascending aortic aneurysm is a vascular pathology when the diameter of the aorta increases more than one and a half [1]. It carries a high risk of dissection and rupture, it should be treated when the size of the aorta exceeds $5.5 \mathrm{~cm}$ for normal individuals, $4,5-5 \mathrm{~cm}$ for Marfan syndrome or who will undergo a cardiac operation for another cause, the increasing rate of the diameter size is $\geq 0.5 \mathrm{~cm} /$ year. On the other hand, when there is dissection or rupture operation should be performed without giving any concern to the diameter of the aorta [3].

Replacement of the ascending aortic aneurysm by a synthetic graft is the standard treatment method all over the world. In 1982, Robecsic et al. tried replacing a well-tailored Dacron vascular graft after excising an oval segment from an ascending aortic aneurysm. This surgical technique had not been accepted by many surgeons [4]. Here in our center, we perform another surgical treatment method of the ascending aortic aneurysms by doing linear plication and external wrapping using a Dacron graft in wellselected patients. We had performed this procedure up to 43 patients [5]. In our technique, we do not pen or excise ascending aorta, we just plicate the major curvature of the aorta to decrease its diameter size to an acceptable range, then wrap the whole aorta by a Dacron graft.

All patients were free from any dissection or rupture, their aortic aneurysms were less than $6.0 \mathrm{~cm}$, and aortic root was not dilated. Also, all of them had not any connective tissue disease such as Marfan syndrome.

This surgical technique was be done in concomitant with other cardiac surgical procedures or as isolated surgery and was performed after releasing cross-clamp on beating heart. We had only one case of isolated ascending aortic aneurysm who was operated on a beating heart without the need for a cardiopulmonary bypass machine [6].

\section{Conclusion:}

We found that by using linear plication and external wrapping surgical technique, the cardiopulmonary bypass and cross-clamp times decreased and the need for blood transfusion was decreased. Most of our patients were observed for long periods and no extra morbidity or mortality due to this procedure has not been recorded.

This surgical technique can be performed safely mainly for elderly patients and who has high morbidity and can be affected negatively by prolonged cross-clamping time.

\section{References}

1. Erbel R, Eggebrecht H. (2006). Aortic dimensions and the risk of dissection. Heart; 92, 137-142.

2. Ozcan AV, Alşalaldeh M, Boysan E, Goksin I. (2013). Ascending Aortic Aneurysm Treatment With Linear Plication, and External Wrapping Technique: Mid-T erm Results. Journal of Cardiac Surgery; Including Mechanical, and Biological Support for the Heart, and Lungs.28, 421-426.

3. Hiratzka LF, Bakris GL, Beckman JA, et al. (2010). ACCF/AHA/ AATS/ ACR/ ASA/SCA/ SCAI/ SIR/ STS/ SVM guidelines for the diagnosis and management of patients with thoracic aortic disease: executive summary: Anesth Analg; 111.279-315.

4. Robicsek F. (1982). A new method to treat fusiform aneurysms of the ascending aorta associated with aortic valve disease: an alternative to radical resection. The Annals of thoracic surgery; 34, 92-94.

5. Mohammad Alsalaldeh, A Vefa Özcan, Şafak Şimşek. (2019). Comparison of Replacement and Linear Plication with External Wrapping Surgical Treatment in Ascending Aortic Aneurysm. Surgery \& Case Studies: Open Access Journal (SCSOAJ) 10.31080/eccy 06.00451.

6. Alşalaldeh M, Özcan, AV. (2019). Isolated Ascending Aortic Aneurysm Treated by Linear Plication, and External Wrapping Via Upper-J-Sternotomy Under Off Pump Technique. EC Cardiology; 6, 01-04. 